\title{
Comparison of rice straw and bamboo stick substrates in periphyton-based carp polyculture systems
}

\author{
Sunila Rai ${ }^{1}$, Yang Yi ${ }^{1,2}$, Md Abdul Wahab ${ }^{3}$, Amrit N Bart ${ }^{1} \&$ James S Diana ${ }^{4}$ \\ ${ }^{1}$ Aquaculture and Aquatic Resources Management, School of Environment, Resources and Development, Asian Institute of \\ Technology, Pathumthani,Thailand \\ ${ }^{2}$ College of Aqua-Life Science and Technology, Shanghai Fisheries University, Shanghai, China \\ ${ }^{3}$ Faculty of Fisheries, Bangladesh Agricultural University, Mymensingh, Bangladesh \\ ${ }^{4}$ School of Natural Resources and Environment, University of Michigan, Ann Arbor, MI, USA
}

Correspondence: Y Yi, College of Aqua-Life Science and Technology, Shanghai Fisheries University, Shanghai, China. E-mail: yiyang@shfu.edu.cn

\begin{abstract}
An experiment was conducted to compare rice straw mat and kanchi (bamboo sticks) as substrates in periphyton-based polyculture systems. The experiment had three treatments: (a) no substrate (control), (b) rice straw as a substrate $\left(3 \times 2.7 \mathrm{~kg}\right.$ pond $\left.{ }^{-1}\right)$ and (c) kanchi as a substrate (390 kanchi pond ${ }^{-1}$ ). Fingerlings $(n=40)$ of rohu, Labeo rohita $(24.5 \pm$ $0.5 \mathrm{~g}$ ); mrigal, Cirrhinus mrigala (25.1 $\pm 0.6 \mathrm{~g})$; catla, Catla catla $(25.8 \pm 0.5 \mathrm{~g})$; common carp, Cyprinus carpio $(27.6 \pm 0.6 \mathrm{~g})$, and silver carp, Hypophthalmichthys molitrix $(30.4 \pm 0.9 \mathrm{~g})$ were stocked at a 3:2:2:2:1 ratio and cultured for 90 days. There were no differences in the number of plankton, periphyton and macro-zoobenthos among the treatments. The total plate count of bacteria was higher in the rice straw treatment (41320 million$\mathrm{cfum}^{-2}$ ) than that in the kanchi treatment $\left(11780\right.$ million cfu m $\left.{ }^{-2}\right)$. Growth and the final mean weight of rohu, catla and common carp were higher in the substrate treatments than those in the control. Rice straw and kanchi treatment, respectively, resulted in $38 \%$ and $47 \%$ higher combined total weight gain over control. Gross margin analysis showed that rice straw treatment resulted in more profit than the control and kanchi treatment. Therefore, rice straw has the potential to be used to increase production in the low-input rural aquaculture.
\end{abstract}

Keywords: rice straw, kanchi, substrate, periphyton-based polyculture system

\section{Introduction}

A variety of biodegradable and non-biodegradable substrates have been used in the past to enhance fish production in periphyton-based aquaculture systems. Among these substrates, bamboo has performed the best (Azim, Verdegem, Rahman, Wahab, Van Dam \& Beveridge 2002b; Van Dam, Beveridge, Azim \& Verdegem 2002) but it is expensive and prohibitive for small-scale rural farmers. There is a need to explore an inexpensive and locally available substrate that enhances fish growth.

Rice straw is relatively low cost and is widely available in farms. It has been used to mitigate turbidity in fish ponds (Yi, Lin \& Diana 2003) and to develop bacterial biofilm and periphyton (Ramesh, Shankar, Mohan \& Varghese 1999). Rice straw used as bundles in tanks increased the growth of rohu (Labeo rohita Hamilton, 1822) and common carp, Cyprinus carpio Linnaeus, 1758 (Ramesh et al. 1999; Mridula, Manissary, Keshavnath, Shankar, Nandesha, \& Rajesh 2005), and fringed lipped carp, Labeo fimbriatus Bloch, 1795 (Mridula, Manissary, Keshavnath, Shankar, Nandesha \& Rajesh 2003) significantly. However, the potential use of rice straw in the carp polyculture pond is yet to be explored.

Commonly available kanchi (bamboo sticks) was reported to increase net fish yield by $78 \%$ in monoculture of Kalbaush, Labeo calbasu Hamilton, 1822 (Wahab, Azim, Ali, Beveridge \& Khan 1999) and by $66 \%$ in polyculture of rohu, catla (Catla catla Hamilton, 1822) and Kalbaush (Azim, Verdegem, Khatun, Wahab, Van Dam \& Beveridge 2002a) compared with 
that in the plankton-based system. These studies indicated that rice straw and kanchi could be alternatives to bamboo. The present experiment was carried out to compare the water quality, abundance of plankton, periphyton, benthos and bacteria, growth of carps and economic returns among a plankton-based carp polyculture system, and two periphyton-based carp polyculture systems using rice straw mats and kanchi, respectively, as substrates.

\section{Materials and methods}

The experiment was conducted in nine $40 \mathrm{~m}^{2}$ $(8 \times 5 \mathrm{~m})$ ponds of $1.5 \mathrm{~m}$ deep at Field Laboratory of Bangladesh Agricultural University (BAU) at Mymensingh for 90 days from February to May 2006. The experiment had three treatments in replications of three: (a) a plankton-based system without a substrate (control) (b) rice straw mat and (c) kanchi. The treatments were allocated randomly to the experimental ponds.

Before placing the substrates, all ponds were drained, dried and limed with $\mathrm{CaO}$ at a rate of $250 \mathrm{~kg} \mathrm{ha}^{-1}$. The ponds were filled to $0.30 \mathrm{~m}$ depth 3 days later. Afterwards, 390 kanchi $(1.5 \mathrm{~cm}$ in diameter and $1.8 \mathrm{~m}$ in length) and three rice straw mats $(2 \times 1 \mathrm{~m})$ were fixed vertically to the bottom. Kanchi added an extra surface area of $20 \mathrm{~m}^{2}$ in each pond (Wahab et al. 1999). The number of rice straw mats per pond was derived from a preliminary study carried out at BAU, which revealed that three rice straw mats $(2 \times 1 \mathrm{~m})$ were optimum for $40 \mathrm{~m}^{2}$ ponds. Rice straw mats were prepared by pressing straw bundles between bamboo splits at a loading rate of $625 \mathrm{~kg} \mathrm{ha}^{-1}$ (dry weight, 12\% moisture). All ponds were filled to $1.10 \mathrm{~m}$ depth and fertilized with urea, triple superphosphate (TSP) and cow dung using BAU fertilization rates of 31,16 and $1250 \mathrm{~kg} \mathrm{ha}^{-1}$ respectively (Wahab et al. 1999). Dissolved oxygen (DO) concentrations in the rice straw treatment ponds were monitored for 2 weeks until DO recovered to $2.1 \mathrm{mg} \mathrm{L}^{-1}$. Then, 40 fingerlings of rohu $(24.5 \pm 0.5 \mathrm{~g})$, mrigal $(25.1 \pm 0.6 \mathrm{~g})$, catla (25.8 \pm $0.5 \mathrm{~g})$, silver carp $(30.4 \pm 0.9 \mathrm{~g})$ and common carp $(27.6 \pm 0.6 \mathrm{~g})$ were stocked at a 3:2:2:2:1 ratio.

Dissolved oxygen, temperature and $\mathrm{pH}$ were measured weekly at 06:00, 18:00 and 06:00 hours the next day. Dissolved oxygen concentrations were measured at three depths: 10,50 and $70 \mathrm{~cm}$ below the water surface. The Secchi disc visibility was monitored weekly at 09:00 h. Composite column water samples were collected monthly at 09:00-10:00 h from three locations of each pond for the analyses of total alkalinity, total ammonia nitrogen (TAN), nitrite-nitrogen, soluble reactive phosphorus (SRP), total phosphorus (TP), chlorophyll $a$, total suspended solids (TSS) and total volatile solids (TVS) (APHA 1980). Total nitrogen (TN) was analysed following Raveh and Avnimelech (1979). Plankton number was estimated following Azim, Wahab, Van Dam, Beveridge and Verdegem (2001a).

Rice straws were cut from three different depths (surface, middle and bottom) of each mat, pooled and wrapped in an aluminium foil for monthly periphyton analyses. Each rice straw sample was transferred to an Erlenmeyer flask containing $50 \mathrm{~mL}$ distilled water and shaken in a mechanical shaker for $3 \mathrm{~h}$ to detach periphytons from the straw surface. After removing periphytons, the straw was dried overnight in an oven at $80^{\circ} \mathrm{C}$ to obtain the dry weight. For sample preservation and taxonomic identification, the above method for planktons was followed. The number of periphyton was estimated using the following formula:

$$
N=(P \times C \times 100) / W
$$

where, $N$ is the number of periphyton units; $P$ is the number of periphyton units counted in 10 random fields of S-R cell; $C$ is the volume of the final concentrated sample $(\mathrm{mL})$; and $\mathrm{W}$ is the weight of rice straw $(\mathrm{g})$.

Periphyton taxa were identified to the genus level using keys from Ward and Whipple (1959), Wetzel (1983) and Bellinger (1992). Dry matter of periphytons was estimated by filtering samples through preweighed and oven-dried Whatman GF/C filter papers and drying for $24 \mathrm{~h}$ in an oven at $105^{\circ} \mathrm{C}$. The ovendried samples were combusted in a Muffle furnace at $550{ }^{\circ} \mathrm{C}$ for $30 \mathrm{~min}$ to obtain the ash content $(\%)$. Chlorophyll $a$ concentration was determined following the standard methods (APHA 1980). Sampling, taxonomic identification, enumeration and estimation of biomass of periphytons in the kanchi were performed as described by Azim, Wahab, Van Dam, Beveridge, Huisman and Verdegem (2001b). Total plate counting of bacteria was performed following APHA (1980). Periphyton number, biomass and bacteria total plate count were estimated based on the pond area for comparisons between treatments.

An Ekman dredge $(15 \times 15 \mathrm{~cm})$ was used to collect macro-zoobenthic organisms from the bottom of each pond monthly. Benthos were identified using a dissecting microscope (CH40RF200 Model, Olympus, Japan) following the keys from Ward and 
Whipple (1959) and Needham and Needham (1962). The number of benthos was estimated following Rahman, Verdegem, Nagelkerke, Wahab, Milstein and Verreth (2006):

$$
N=(Y \times 10000) / 3 A
$$

where, $N$ is the number of benthic organisms (individuals $\mathrm{m}^{-2}$ ); $Y$ is the total number of benthic organisms counted in three samples; and $A$ is the area of Ekman dredge $\left(\mathrm{cm}^{2}\right)$.

At the end, substrates were removed from the ponds, and fish were harvested, counted and weighed individually to estimate weight gains and survival rates. Gross margin analysis was carried out to determine economic returns for the three treatments. The local market prices of all inputs and outputs in Mymensingh of Bangladesh were used in the economic analysis. The analysis excluded labour cost as rural farmers use family labours to get farm work done. Expectant life of bamboo and kanchi was assumed to be 3 and 1.5 years, respectively.

Water quality, plankton, fish growth and economic parameters were compared by one-way analysis of variance (ANOVA) using SPSS (version 12.0) statistical software (SPSS, Chicago, IL, USA). A Tukey's test was performed if significant differences were found by ANOVA. Periphyton and bacteria were compared by Student's $t$-test. Differences were considered significant at an $\alpha$ level of $0.05(P<0.05)$. All means were given with $\pm 1 \mathrm{SD}$.

\section{Results}

All water quality parameters, except DO, did not differ among all treatments (Table 1). Dissolved oxygen concentrations at 06:00 and 18:00 hours were higher in the control and the kanchi treatment than those in the rice straw treatment at all three depths.

There were no differences in the densities of phytoplankton and zooplankton among the treatments (Tables 2 and 3). The abundance of macrozoobenthos in pond sediment was $361 \pm 101$, $320 \pm 104$ and $275 \pm 90$ individual $\mathrm{m}^{-2}$ in the control, rice straw and kanchi treatments, respectively.

There were no significant differences in the densities of periphyton between the rice straw and kanchi treatments (Table 4). Dry matter, ash and chlorophyll a content of periphyton also did not differ between the rice straw and kanchi treatments. However,

Table 1 Overall mean values $( \pm S D$ ) of water quality parameters measured throughout the experiment

\begin{tabular}{|c|c|c|c|}
\hline \multirow[b]{2}{*}{ Water-quality parameters } & \multicolumn{3}{|l|}{ Treatments } \\
\hline & Control & Rice straw & Kanchi \\
\hline Temperature at $06: 00$ hours $\left({ }^{\circ} \mathrm{C}\right)$ & $25.8 \pm 0.6$ & $25.9 \pm 0.5$ & $26.1 \pm 0.1$ \\
\hline Temperature at $18: 00$ hours $\left({ }^{\circ} \mathrm{C}\right)$ & $29.3 \pm 0.1$ & $29.4 \pm 0.1$ & $29.3 \pm 0.1$ \\
\hline \multicolumn{4}{|l|}{ DO at $06: 00$ hours $\left(\mathrm{mg} \mathrm{L}^{-1}\right)$} \\
\hline $10 \mathrm{~cm}$ & $5.3 \pm 0.1^{a}$ & $3.7 \pm 0.1^{\mathrm{b}}$ & $4.9 \pm 0.1^{\mathrm{a}}$ \\
\hline $50 \mathrm{~cm}$ & $4.6 \pm 0.3^{a}$ & $3.2 \pm 0.1^{\mathrm{b}}$ & $4.2 \pm 0.2^{\mathrm{a}}$ \\
\hline $70 \mathrm{~cm}$ & $4.0 \pm 0.3^{\mathrm{a}}$ & $2.7 \pm 0.1^{\mathrm{b}}$ & $3.5 \pm 0.2^{\mathrm{a}}$ \\
\hline \multicolumn{4}{|l|}{$\mathrm{DO}$ at $18: 00$ hours $\left(\mathrm{mg} \mathrm{L}^{-1}\right)$} \\
\hline $10 \mathrm{~cm}$ & $9.3 \pm 0.5^{a}$ & $7.5 \pm 0.3^{b}$ & $9.2 \pm 0.3^{\mathrm{a}}$ \\
\hline $50 \mathrm{~cm}$ & $7.4 \pm 0.5^{\mathrm{a}}$ & $5.8 \pm 0.3^{\mathrm{b}}$ & $7.8 \pm 0.8^{\mathrm{a}}$ \\
\hline $70 \mathrm{~cm}$ & $5.9 \pm 0.4^{a}$ & $4.8 \pm 0.3^{b}$ & $5.9 \pm 0.2^{\mathrm{a}}$ \\
\hline $\mathrm{pH}$ at $06: 00$ hours & $8.5 \pm 0.0$ & $8.4 \pm 0.0$ & $8.5 \pm 0.0$ \\
\hline $\mathrm{pH}$ at $18: 00$ hours & $8.9 \pm 0.0$ & $8.7 \pm 0.0$ & $8.9 \pm 0.0$ \\
\hline Secchi disc depth $(\mathrm{cm})$ & $18.2 \pm 0.7$ & $21.6 \pm 2.6$ & $20.5 \pm 0.6$ \\
\hline Total alkalinity $\left(\mathrm{mg} \mathrm{L}^{-1}\right.$ as $\left.\mathrm{CaCO}_{3}\right)$ & $128 \pm 13$ & $138 \pm 8$ & $129 \pm 8$ \\
\hline Chlorophyll a $\left(\mu \mathrm{g} \mathrm{L}^{-1}\right)$ & $56 \pm 11$ & $48 \pm 20$ & $61 \pm 23$ \\
\hline Total nitrogen $\left(\mathrm{mg} \mathrm{L}^{-1}\right)$ & $1.43 \pm 0.45$ & $1.53 \pm 0.59$ & $1.60 \pm 0.17$ \\
\hline Total ammonia nitrogen $\left(\mathrm{mg} \mathrm{L}^{-1}\right)$ & $0.17 \pm 0.14$ & $0.10 \pm 0.03$ & $0.14 \pm 0.07$ \\
\hline Nitrite nitrogen $\left(\mathrm{mg} \mathrm{L}^{-1}\right)$ & $0.01 \pm 0.00$ & $0.01 \pm 0.00$ & $0.01 \pm 0.01$ \\
\hline Total phosphorous ( $\mathrm{mgL}^{-1}$ ) & $2.30 \pm 0.46$ & $1.73 \pm 0.15$ & $1.83 \pm 0.23$ \\
\hline Soluble reactive phosphorous $\left(\mathrm{mg} \mathrm{L}^{-1}\right)$ & $2.30 \pm 0.78$ & $1.03 \pm 0.21$ & $1.53 \pm 0.91$ \\
\hline Total suspended solids $\left(\mathrm{mg} \mathrm{L}^{-1}\right)$ & $75 \pm 20$ & $70 \pm 6$ & $58 \pm 11$ \\
\hline Total volatile solids $\left(\mathrm{mg} \mathrm{L}^{-1}\right)$ & $43 \pm 14$ & $39 \pm 4$ & $32 \pm 12$ \\
\hline
\end{tabular}

Mean values with different superscript letters in the same row are significantly different $(P<0.05)$.

DO, dissolved oxygen. 
Table 2 Abundance (Mean \pm SD) of phytoplankton in the pond water in different treatments

\begin{tabular}{|c|c|c|c|c|}
\hline \multirow[b]{2}{*}{ Group } & \multirow[b]{2}{*}{ Genus } & \multicolumn{3}{|l|}{ Treatments } \\
\hline & & Control (units $\mathrm{L}^{-1}$ ) & Rice straw (units $\mathrm{L}^{-1}$ ) & Kanchi (units $\mathrm{L}^{-1}$ ) \\
\hline \multirow[t]{13}{*}{ Bacillariophyceae } & Coscinodiscus & $2457 \pm 827$ & $3405 \pm 2448$ & $4233 \pm 4639$ \\
\hline & Cyclotella & $6145 \pm 2803$ & $26418 \pm 21938$ & $9000 \pm 4639$ \\
\hline & Cymbella & $67 \pm 115$ & $0 \pm 0$ & $0 \pm 0$ \\
\hline & Diatoma & $257 \pm 283$ & $303 \pm 316$ & $250 \pm 250$ \\
\hline & Gomphonema & $512 \pm 310$ & $473 \pm 231$ & $597 \pm 650$ \\
\hline & Melosira & $6310 \pm 7358$ & $1477 \pm 898$ & $2510 \pm 2181$ \\
\hline & Fragillaria & $2005 \pm 841$ & $1765 \pm 1071$ & $1348 \pm 619$ \\
\hline & Navicula & $1875 \pm 1797$ & $1390 \pm 863$ & $2127 \pm 1039$ \\
\hline & Nitzschia & $4853 \pm 2254$ & $2967 \pm 1432$ & $17970 \pm 21136$ \\
\hline & Surirella & $575 \pm 532$ & $1047 \pm 464$ & $700 \pm 889$ \\
\hline & Synedra & $1953 \pm 1649$ & $2080 \pm 719$ & $1345 \pm 650$ \\
\hline & Tabellaria & $173 \pm 152$ & $322 \pm 376$ & $167 \pm 289$ \\
\hline & Subtotal & $27182 \pm 9662$ & $41647 \pm 21498$ & $40247 \pm 20160$ \\
\hline \multirow[t]{25}{*}{ Chlorophyceae } & Actinastrum & $412 \pm 523$ & $1062 \pm 917$ & $1302 \pm 1512$ \\
\hline & Ankistrodesmus & $423 \pm 405$ & $743 \pm 1094$ & $597 \pm 406$ \\
\hline & Centritractus & $78 \pm 136$ & $0 \pm 0$ & $1000 \pm 1000$ \\
\hline & Chlamydomonas & $0 \pm 0$ & $513 \pm 208$ & $710 \pm 648$ \\
\hline & Chlorella & $13087 \pm 9901$ & $12305 \pm 6053$ & $57668 \pm 84742$ \\
\hline & Chodatella & $228 \pm 395$ & $0 \pm 0$ & $0 \pm 0$ \\
\hline & Closterium & $125 \pm 109$ & $1557 \pm 1939$ & $1017 \pm 1087$ \\
\hline & Coelastrum & $1253 \pm 1846$ & $712 \pm 798$ & $472 \pm 640$ \\
\hline & Crucigenia & $4692 \pm 4356$ & $3843 \pm 3761$ & $1696 \pm 838$ \\
\hline & Gonatozygon & $350 \pm 312$ & $72 \pm 124$ & $375 \pm 327$ \\
\hline & Mougeotia & $2225 \pm 2927$ & $2798 \pm 2325$ & $1440 \pm 1276$ \\
\hline & Oedogonium & $0 \pm 0$ & $72 \pm 124$ & $167 \pm 288$ \\
\hline & Oocystis & $6695 \pm 1858$ & $4483 \pm 580$ & $17107 \pm 10501$ \\
\hline & Pediastrum & $2598 \pm 1440$ & $933 \pm 275$ & $2127 \pm 1056$ \\
\hline & Scenedesmus & $9593 \pm 7694$ & $10213 \pm 5375$ & $8082 \pm 4720$ \\
\hline & Selenastrum & $268 \pm 263$ & $140 \pm 242$ & $2832 \pm 4673$ \\
\hline & Sphaerocystis & $5457 \pm 5924$ & $2253 \pm 998$ & $10047 \pm 9486$ \\
\hline & Spirogyra & $67 \pm 115$ & $0 \pm 0$ & $0 \pm 0$ \\
\hline & Staurastrum & $95 \pm 165$ & $212 \pm 210$ & $83 \pm 144$ \\
\hline & Tetraedron & $543 \pm 587$ & $503 \pm 472$ & $250 \pm 433$ \\
\hline & Tetraspora & $290 \pm 254$ & $783 \pm 916$ & $313 \pm 372$ \\
\hline & Treubaria & $95 \pm 165$ & $233 \pm 214$ & $167 \pm 289$ \\
\hline & Ulothrix & $1025 \pm 631^{a}$ & $142 \pm 123^{b}$ & $142 \pm 128^{b}$ \\
\hline & Volvox & $75 \pm 130$ & $70 \pm 121$ & $167 \pm 287$ \\
\hline & Subtotal & $49675 \pm 21291$ & $43643 \pm 10318$ & $107759 \pm 99027$ \\
\hline \multirow[t]{10}{*}{ Cyanophyceae } & Anabaena & $75 \pm 130$ & $0 \pm 0$ & $0 \pm 0$ \\
\hline & Aphanocapsa sp. & $0 \pm 0$ & $140 \pm 242$ & $167 \pm 289$ \\
\hline & Aphanozomenon & $0 \pm 0$ & $75 \pm 130$ & $0 \pm 0$ \\
\hline & Chroococcus & $1527 \pm 1233$ & $2445 \pm 599$ & $1633 \pm 1886$ \\
\hline & Gloecapsa & $1507 \pm 1754$ & $952 \pm 303$ & $1380 \pm 1777$ \\
\hline & Gomphosphaeria & $275 \pm 152$ & $238 \pm 254$ & $1325 \pm 1211$ \\
\hline & Merismopedia & $467 \pm 808$ & $528 \pm 162$ & $0 \pm 0$ \\
\hline & Microcystis & $78 \pm 136$ & $70 \pm 121$ & $0 \pm 0$ \\
\hline & Oscillatoria & $875 \pm 1277$ & $412 \pm 375$ & $400 \pm 529$ \\
\hline & Subtotal & $4803 \pm 1309$ & $4860 \pm 765$ & $4905 \pm 4773$ \\
\hline \multirow[t]{4}{*}{ Euglenophyceae } & Euglena & $9678 \pm 4937$ & $41310 \pm 51261$ & $88363 \pm 108714$ \\
\hline & Phacus & $548 \pm 472$ & $1495 \pm 676$ & $672 \pm 70$ \\
\hline & Trachalomonas & $1447 \pm 596$ & $1672 \pm 1285$ & $1298 \pm 1202$ \\
\hline & Subtotal & $11673 \pm 5470$ & $44477 \pm 49373$ & $90333 \pm 107749$ \\
\hline Total phytoplankton & & $93333 \pm 15342$ & $134627 \pm 62439$ & $243244 \pm 94646$ \\
\hline Number of identified genus & & 45 & 44 & 42 \\
\hline
\end{tabular}

Mean values with different superscript letters in the same row are significantly different $(P<0.05)$. 
Table 3 Abundance (Mean \pm SD) of zooplankton in the pond water in different treatments

\begin{tabular}{|c|c|c|c|c|}
\hline \multirow[b]{2}{*}{ Group } & \multirow[b]{2}{*}{ Genus } & \multicolumn{3}{|l|}{ Treatments } \\
\hline & & Control (units $\mathrm{L}^{-1}$ ) & Rice straw (units $\mathrm{L}^{-1}$ ) & Kanchi (units $L^{-1}$ ) \\
\hline Sarcodina & Difflusia & $962 \pm 523$ & $820 \pm 589$ & $1678 \pm 1458$ \\
\hline \multirow[t]{9}{*}{ Rotifera } & Asplanchna & $740 \pm 275$ & $837 \pm 609$ & $1833 \pm 1318$ \\
\hline & Brachionus & $1343 \pm 534$ & $2027 \pm 178$ & $4477 \pm 2840$ \\
\hline & Filinia & $1278 \pm 912$ & $227 \pm 393$ & $337 \pm 111$ \\
\hline & Keratella & $1577 \pm 932$ & $1153 \pm 73$ & $1260 \pm 176$ \\
\hline & Lecane & $200 \pm 25$ & $445 \pm 438$ & $575 \pm 378$ \\
\hline & Monostylla & $0 \pm 0$ & $0 \pm 0$ & $250 \pm 250$ \\
\hline & Polyarthra & $1055 \pm 989$ & $987 \pm 309$ & $2013 \pm 533$ \\
\hline & Trichocerca & $577 \pm 327$ & $150 \pm 130$ & $330 \pm 361$ \\
\hline & Subtotal & $6848 \pm 2044$ & $5895 \pm 703$ & $11075 \pm 4133$ \\
\hline \multirow[t]{8}{*}{ Crustacea } & Cyclpos & $545 \pm 135$ & $1068 \pm 293$ & $590 \pm 512$ \\
\hline & Diaptomus & $235 \pm 41$ & $148 \pm 129$ & $303 \pm 366$ \\
\hline & Ceriodaphnia & $58 \pm 101$ & $130 \pm 114$ & $600 \pm 529$ \\
\hline & Daphnia & $75 \pm 130$ & $338 \pm 118$ & $0 \pm 0$ \\
\hline & Diaphanosoma & $290 \pm 182$ & $87 \pm 150$ & $67 \pm 115$ \\
\hline & Moina & $240 \pm 243$ & $450 \pm 507$ & $553 \pm 224$ \\
\hline & Nauplius & $772 \pm 130$ & $1308 \pm 591$ & $1215 \pm 133$ \\
\hline & Subtotal & $2215 \pm 362$ & $3530 \pm 563$ & $3328 \pm 897$ \\
\hline Total zooplankton & & $9947 \pm 1379$ & $10176 \pm 1158$ & $16082 \pm 4089$ \\
\hline Number of identified genus & & 15 & 15 & 15 \\
\hline
\end{tabular}

Table 4 Abundance (Mean \pm SD) of periphyton in terms of the pond surface area in the rice straw and kanchi treatments during the experimental period

\begin{tabular}{|c|c|c|c|}
\hline \multirow[b]{2}{*}{ Group } & \multirow[b]{2}{*}{ Genus } & \multicolumn{2}{|l|}{ Treatments } \\
\hline & & Rice straw $\left(10^{3} \times{\left.\text { units } \mathrm{m}^{-2}\right)}^{-2}\right.$ & Kanchi $\left(10^{3} \times{\left.\text { units } \mathrm{m}^{-2}\right)}^{-2}\right.$ \\
\hline \multirow[t]{13}{*}{ Bacillariophyceae } & Coscinodiscus & $794 \pm 1376$ & $0 \pm 0$ \\
\hline & Cyclotella & $3720 \pm 1182^{a}$ & $174 \pm 301^{\mathrm{b}}$ \\
\hline & Cymbella & $2863 \pm 2481$ & $0 \pm 0$ \\
\hline & Diatoma & $34999 \pm 16623^{a}$ & $3991 \pm 795^{\mathrm{b}}$ \\
\hline & Fragillaria & $15931 \pm 7163$ & $5553 \pm 3347$ \\
\hline & Gomphonema & $7680 \pm 2959^{a}$ & $521 \pm 521^{\mathrm{b}}$ \\
\hline & Melosira & $7095 \pm 3932$ & $868 \pm 795$ \\
\hline & Navicula & $40196 \pm 47396$ & $13362 \pm 7832$ \\
\hline & Nitzschia & $64369 \pm 33405^{a}$ & $6594 \pm 1828^{b}$ \\
\hline & Surirella & $1589 \pm 2751$ & $0 \pm 0$ \\
\hline & Synedra & $30476 \pm 12967$ & $12147 \pm 9547$ \\
\hline & Tabellaria & $1838 \pm 1635$ & $174 \pm 301$ \\
\hline & Subtotal & $211550 \pm 30722^{a}$ & $43382 \pm 6254^{b}$ \\
\hline \multirow[t]{13}{*}{ Chlorophyceae } & Actinastrum & $531 \pm 919$ & $0 \pm 0$ \\
\hline & Centritractus & $1592 \pm 2757$ & $0 \pm 0$ \\
\hline & Characium & $2196 \pm 2109$ & $3471 \pm 2568$ \\
\hline & Chlamydomonas & $0 \pm 0$ & $174 \pm 301$ \\
\hline & Chlorella & $40049 \pm 7860^{a}$ & $5900 \pm 3837^{b}$ \\
\hline & Closterium & $1805 \pm 3126$ & $0 \pm 0$ \\
\hline & Coelastrum & $0 \pm 0$ & $347 \pm 601$ \\
\hline & Cosmarium & $5045 \pm 2497$ & $1215 \pm 301$ \\
\hline & Crucigenia & $37428 \pm 26208$ & $1562 \pm 1377$ \\
\hline & Cylindrocapsa & $0 \pm 0$ & $22038 \pm 22866$ \\
\hline & Gonatozygon & $4822 \pm 2329$ & $0 \pm 0$ \\
\hline & Microspora & $3839 \pm 582$ & $1041 \pm 1803$ \\
\hline & Mougeotia & $7095 \pm 7046$ & $868 \pm 1084$ \\
\hline
\end{tabular}


Table 4 Continued

\begin{tabular}{|c|c|c|c|}
\hline \multirow[b]{2}{*}{ Group } & \multirow[b]{2}{*}{ Genus } & \multicolumn{2}{|l|}{ Treatments } \\
\hline & & 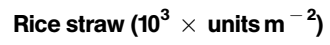 & Kanchi $\left(10^{3} \times\right.$ units $\left.^{-2}\right)$ \\
\hline & Oedogonium & $5420 \pm 7421$ & $9718 \pm 7969$ \\
\hline & Oocystis & $3592 \pm 3892$ & $4165 \pm 1562$ \\
\hline & Scenedesmus & $60020 \pm 15990^{a}$ & $5206 \pm 521^{b}$ \\
\hline & Stigeoclonium & $1589 \pm 2751$ & $476160 \pm 251769$ \\
\hline & Sphaerocystis & $720 \pm 1248$ & $0 \pm 0$ \\
\hline & Staurastrum & $1016 \pm 1760$ & $0 \pm 0$ \\
\hline & Tetraspora & $0 \pm 0$ & $694 \pm 301$ \\
\hline & Tetradorn & $0 \pm 0$ & $1041 \pm 1377$ \\
\hline & Triplocerus & $5384 \pm 2958$ & $0 \pm 0$ \\
\hline & Ulothrix & $0 \pm 0$ & $1735 \pm 1590$ \\
\hline & Subtotal & $182141 \pm 27784$ & $535332 \pm 238794$ \\
\hline \multirow[t]{7}{*}{ Cyanophyceae } & Chroococcus & $4800 \pm 6980$ & $2082 \pm 902$ \\
\hline & Gleocapsa & $0 \pm 0$ & $3991 \pm 1084$ \\
\hline & Gomphosphaeria & $1203 \pm 1064$ & $0 \pm 0$ \\
\hline & Merismopedia & $1044 \pm 1808$ & $0 \pm 0$ \\
\hline & Oscillatoria & $19493 \pm 12661$ & $2429 \pm 1202$ \\
\hline & Phormidium & $25304 \pm 13958^{\mathrm{a}}$ & $1215 \pm 1673^{b}$ \\
\hline & Subtotal & $51844 \pm 24230$ & $9718 \pm 601$ \\
\hline \multirow[t]{4}{*}{ Euglenophyceae } & Euglena & $5426 \pm 3492$ & $1388 \pm 1084$ \\
\hline & Phacus & $720 \pm 1248$ & $0 \pm 0$ \\
\hline & Trachelomonas & $2842 \pm 2462$ & $174 \pm 301$ \\
\hline & Subtotal & $8989 \pm 1293^{a}$ & $1562 \pm 1377^{b}$ \\
\hline Sarcodina & Diffugia & $531 \pm 919$ & $0 \pm 0$ \\
\hline \multirow[t]{6}{*}{ Rotifera } & Asplanchna & $1589 \pm 2751$ & $694 \pm 301$ \\
\hline & Brachionus & $1838 \pm 1635$ & $521 \pm 521$ \\
\hline & Conochilus & $0 \pm 0$ & $1562 \pm 902$ \\
\hline & Lecane & $1346 \pm 2331$ & $0 \pm 0$ \\
\hline & Subtotal & $4773 \pm 2107$ & $2776 \pm 795$ \\
\hline & Total periphyton & $459826 \pm 32266$ & $592770 \pm 233709$ \\
\hline Number of identified genus & & 41 & 34 \\
\hline
\end{tabular}

Mean values with different superscript letters in the same row are significantly different $(P<0.05)$.

Table 5 Periphyton biomass (Mean \pm SD) in the rice straw and kanchi treatments

\begin{tabular}{lcc}
\hline & \multicolumn{2}{l}{ Treatment } \\
\cline { 2 - 3 } Parameter & Rice straw & Kanchi \\
\hline Dry matter $\left(\right.$ g pond $^{-1}$ ) & $540.4 \pm 140.6$ & $620.3 \pm 60.1$ \\
Ash (\%) & $41.7 \pm 3.7$ & $36.9 \pm 7.3$ \\
Ash-free dry matter $\left(\right.$ g pond $^{-1}$ ) & $280.0 \pm 36.6^{\mathrm{b}}$ & $382.8 \pm 36.8^{\mathrm{a}}$ \\
${\text { Chlorophyll } a \text { (g pond }^{-1} \text { ) }}$ & $0.75 \pm 0.2$ & $2.18 \pm 0.9$ \\
\hline
\end{tabular}

Mean values with different superscript letters in the same row are significantly different $(P<0.05)$.

ash-free dry matter was higher in the kanchi treatment than in the rice straw treatment (Table 5). Bacteria total plate count was $41320 \pm 4006$ $\left(10^{6} \times \mathrm{cfu} \mathrm{m}^{-2}\right)$ on the rice straw, which was higher than $11780 \pm 1208\left(10^{6} \times \mathrm{cfu} \mathrm{m}^{-2}\right)$ on the kanchi.
Treatments with substrates had higher fish growth (Table 6). The final size of individuals and daily weight gains of rohu and common carp were higher in the substrate treatments than in the control. There was no difference between the two-substrate treatments. The final size and daily weight gain of catla were higher in the kanchi treatment than that in the control but were not different from the rice straw treatment. The total weight gains of rohu and catla were higher in the substrate treatments than those in the control, although there were no differences in total weight gains of mrigal, common carp and silver carp among treatments. Furthermore, individual final size and daily weight gains of mrigal and silver carp were not different among treatments. Combined total weight gain was significantly higher in the substrate treatments than that in the control but there were no differences between the twosubstrate treatments. Silver carp made the highest 
Table 6 Growth performance of carps stocked in different treatments

\begin{tabular}{|c|c|c|c|c|c|c|}
\hline Treatments & Rohu & Catla & Mrigal & Common carp & Silver carp & Combined \\
\hline \multicolumn{7}{|c|}{ Initial total weight $\left(\mathrm{kgpond}^{-1}\right)$} \\
\hline Control & $0.30 \pm 0.01$ & $0.20 \pm 0.01$ & $0.20 \pm 0.01$ & $0.22 \pm 0.01$ & $0.13 \pm 0.01$ & $1.05 \pm 0.04$ \\
\hline Rice straw & $0.28 \pm 0.02$ & $0.21 \pm 0.01$ & $0.21 \pm 0.02$ & $0.21 \pm 0.01$ & $0.11 \pm 0.01$ & $1.01 \pm 0.03$ \\
\hline Kanchi & $0.30 \pm 0.01$ & $0.21 \pm 0.02$ & $0.19 \pm 0.01$ & $0.23 \pm 0.00$ & $0.13 \pm 0.01$ & $1.07 \pm 0.01$ \\
\hline \multicolumn{7}{|c|}{ Initial mean weight ( $\mathrm{g} \mathrm{fish}^{-1}$ ) } \\
\hline Control & $25.10 \pm 0.79$ & $25.50 \pm 1.39$ & $24.83 \pm 1.76$ & $27.90 \pm 1.04$ & $31.40 \pm 2.07$ & \\
\hline Rice straw & $23.17 \pm 1.63$ & $25.83 \pm 1.37$ & $26.13 \pm 2.63$ & $25.67 \pm 1.44$ & $28.53 \pm 2.32$ & \\
\hline Kanchi & $25.33 \pm 1.06$ & $26.03 \pm 2.42$ & $24.33 \pm 1.08$ & $29.23 \pm 0.21$ & $31.43 \pm 3.13$ & \\
\hline \multicolumn{7}{|c|}{ Final total weight (kg pond $\left.{ }^{-1}\right)$} \\
\hline Control & $1.19 \pm 0.06^{b}$ & $0.43 \pm 0.05^{\mathrm{b}}$ & $0.89 \pm 0.13$ & $0.58 \pm 0.06$ & $1.35 \pm 0.20$ & $4.44 \pm 0.36^{b}$ \\
\hline Rice straw & $1.66 \pm 0.09^{a}$ & $0.63 \pm 0.04^{a}$ & $1.16 \pm 0.11$ & $0.71 \pm 0.08$ & $1.53 \pm 0.03$ & $5.70 \pm 0.06^{\mathrm{a}}$ \\
\hline Kanchi & $1.90 \pm 0.27^{\mathrm{a}}$ & $0.73 \pm 0.06^{a}$ & $1.19 \pm 0.26$ & $0.64 \pm 0.09$ & $1.60 \pm 0.20$ & $6.06 \pm 0.70^{\mathrm{a}}$ \\
\hline \multicolumn{7}{|c|}{ Final mean weight $\left(\mathrm{g} \mathrm{fish}^{-1}\right.$ ) } \\
\hline Control & $105.41 \pm 0.89^{b}$ & $59.00 \pm 10.36^{\mathrm{b}}$ & $130.49 \pm 7.25$ & $72.67 \pm 8.02^{\mathrm{b}}$ & $406.19 \pm 32.23$ & \\
\hline Rice straw & $138.72 \pm 7.60^{\mathrm{a}}$ & $68.67 \pm 21.36^{\mathrm{ab}}$ & $139.81 \pm 38.70$ & $90.35 \pm 5.41^{\mathrm{a}}$ & $365.71 \pm 18.78$ & \\
\hline Kanchi & $162.82 \pm 16.77^{a}$ & $99.37 \pm 9.30^{\mathrm{a}}$ & $161.15 \pm 18.88$ & $92.01 \pm 2.53^{a}$ & $402.46 \pm 51.82$ & \\
\hline \multicolumn{7}{|c|}{ Daily weight gain (g fish ${ }^{-1}$ day $^{-1}$ ) } \\
\hline Control & $0.89 \pm 0.00^{\mathrm{b}}$ & $0.37 \pm 0.13^{b}$ & $1.17 \pm 0.08$ & $0.50 \pm 0.08^{\mathrm{b}}$ & $4.16 \pm 0.34$ & \\
\hline Rice straw & $1.28 \pm 0.07^{\mathrm{a}}$ & $0.48 \pm 0.22^{\mathrm{ab}}$ & $1.26 \pm 0.40$ & $0.72 \pm 0.05^{\mathrm{a}}$ & $3.75 \pm 0.18$ & \\
\hline Kanchi & $1.53 \pm 0.18^{\mathrm{a}}$ & $0.81 \pm 0.12^{\mathrm{a}}$ & $1.52 \pm 0.22$ & $0.70 \pm 0.03^{\mathrm{a}}$ & $4.12 \pm 0.55$ & \\
\hline \multicolumn{7}{|c|}{ Total weight gain (kg pond ${ }^{-1}$ ) } \\
\hline Control & $0.89 \pm 0.06^{b}$ & $0.23 \pm 0.06^{b}$ & $0.69 \pm 0.12$ & $0.36 \pm 0.06$ & $1.22 \pm 0.19$ & $3.39 \pm 0.32^{b}$ \\
\hline Rice straw & $1.39 \pm 0.08^{\mathrm{a}}$ & $0.42 \pm 0.04^{a}$ & $0.96 \pm 0.08$ & $0.50 \pm 0.08$ & $1.44 \pm 0.04$ & $4.69 \pm 0.06^{a}$ \\
\hline Kanchi & $1.60 \pm 0.26^{a}$ & $0.52 \pm 0.08^{a}$ & $0.99 \pm 0.27$ & $0.41 \pm 0.09$ & $1.47 \pm 0.19$ & $4.99 \pm 0.70^{a}$ \\
\hline \multicolumn{7}{|l|}{ Survival (\%) } \\
\hline Control & $94.7 \pm 4.6$ & $96.0 \pm 6.9$ & $96.0 \pm 6.9$ & $100.0 \pm 0.0$ & $83.0 \pm 14.4$ & \\
\hline Rice straw & $100.0 \pm 0.0$ & $96.0 \pm 6.9$ & $96.0 \pm 6.9$ & $96.0 \pm 6.9$ & $100.0 \pm 0.0$ & \\
\hline Kanchi & $97.3 \pm 4.6$ & $92.0 \pm 6.9$ & $91.7 \pm 14.4$ & $87.7 \pm 12.5$ & $100.0 \pm 0.0$ & \\
\hline
\end{tabular}

Mean values with different superscript letters in the same column among treatments are significantly different $(P<0.05)$.

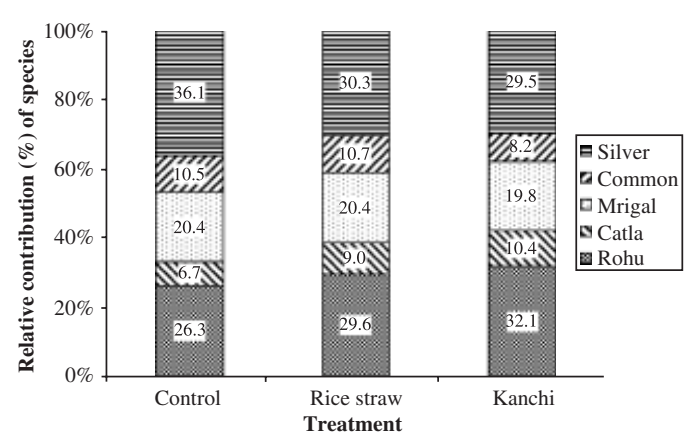

Figure 1 Relative contribution (\%) of five species on combined total weight gains in different treatments.

contribution (30-36\%) to the total weight gains in all three treatments although they represented only $10 \%$ of the total population, while catla contributed the least $(7-10 \%)$. Rohu, mrigal and common carp contributed $26-32 \%, 20 \%$ and $8-11 \%$, respectively, to the total weight gains (Fig. 1). There were no differences in the survival rates among all five species across treatments $(P>0.05)$.

Gross margin analysis showed that all treatments were profitable; however, there was no significant difference in gross margin among all treatments (Table 7).

\section{Discussion}

Generally, water quality remained within the normal range during the experiment. Significantly lower DO in the rice straw treatment was probably due to increased biological oxygen demand (Dharmaraj, Manissery \& Keshavnath 2002), which is common in the water with predominantly heterotrophic food production (Moriarty 1997).

Plankton density did not differ among all treatments, indicating that the added substrates did not affect plankton growth. Lower ash-free dry matter of periphyton in the rice straw treatment than that in 
Table 7 Gross margin analysis of different treatments based on $40 \mathrm{~m}^{2}$ pond in Bangladeshi currency Taka (1 US\$ $=67$ Taka)

\begin{tabular}{|c|c|c|c|c|c|c|c|c|}
\hline \multirow[b]{3}{*}{ Item } & \multicolumn{8}{|c|}{ Treatments } \\
\hline & \multirow[b]{2}{*}{ Unit } & \multirow[b]{2}{*}{ Taka/unit } & \multicolumn{2}{|l|}{ Control } & \multicolumn{2}{|l|}{ Rice straw } & \multicolumn{2}{|l|}{ Kanchi } \\
\hline & & & Quantity & Taka & Quantity & Taka & Quantity & Taka \\
\hline \multicolumn{9}{|l|}{ Gross return } \\
\hline Rohu & $\mathrm{kg}$ & 60 & 1.19 & $72 \pm 4^{\mathrm{b}}$ & 1.66 & $100 \pm 5^{a}$ & 1.90 & $114 \pm 16^{\mathrm{a}}$ \\
\hline Catla & $\mathrm{kg}$ & 60 & 0.43 & $26 \pm 3^{b}$ & 0.63 & $38 \pm 2^{a}$ & 0.73 & $44 \pm 3^{\mathrm{a}}$ \\
\hline Mrigal & $\mathrm{kg}$ & 60 & 0.89 & $53 \pm 8$ & 1.16 & $70 \pm 6$ & 1.19 & $71 \pm 16$ \\
\hline Common & $\mathrm{kg}$ & 60 & 0.58 & $35 \pm 4$ & 0.71 & $42 \pm 5$ & 0.64 & $39 \pm 5$ \\
\hline Silver & $\mathrm{kg}$ & 60 & 1.35 & $81 \pm 12$ & 1.53 & $92 \pm 2$ & 1.60 & $96 \pm 12$ \\
\hline Total gross return & & & & $267 \pm 21^{b}$ & & $342 \pm 4^{a}$ & & $364 \pm 42^{\mathrm{a}}$ \\
\hline \multicolumn{9}{|l|}{ Variable cost } \\
\hline \multicolumn{9}{|l|}{ Fingerlings } \\
\hline Rohu & Pcs & 3.5 & 12 & 42 & 12 & 42 & 12 & 42 \\
\hline Catla & Pcs & 3.5 & 8 & 28 & 8 & 28 & 8 & 28 \\
\hline Mrigal & Pcs & 3.5 & 8 & 28 & 8 & 28 & 8 & 28 \\
\hline Common & Pcs & 3.5 & 8 & 28 & 8 & 28 & 8 & 28 \\
\hline Silver & Pcs & 3.5 & 4 & 14 & 4 & 14 & 4 & 14 \\
\hline \multicolumn{9}{|l|}{ Fertilizer } \\
\hline Urea & $\mathrm{kg}$ & 8 & 0.9 & 7 & 0.9 & 7 & 0.9 & 7 \\
\hline TSP & $\mathrm{kg}$ & 15 & 0.4 & 7 & 0.4 & 7 & 0.4 & 7 \\
\hline Cow dung & $\mathrm{kg}$ & 0.4 & 35.0 & 14 & 35.0 & 14 & 35.0 & 14 \\
\hline Lime & $\mathrm{kg}$ & 12 & 1.0 & 12 & 1.0 & 12 & 1.0 & 12 \\
\hline Kanchi & Pcs & 1 & & - & - & - & 390 for 4 crops & 98 \\
\hline Bamboo & Pcs & 130 & - & - & 2 for 9 crops & 29 & - & - \\
\hline Wire & & & & & & 20 & & \\
\hline Interest on working capital & & $10 \%$ & & 4 & & 6 & & 7 \\
\hline Total variable cost & & & & $184 \pm 0$ & & $235 \pm 0$ & & $285 \pm 0$ \\
\hline Gross margin & & & & $83 \pm 21$ & & $107 \pm 4$ & & $79 \pm 42$ \\
\hline
\end{tabular}

Mean values with different superscript letters in the same row are significantly different $(P<0.05)$.

TSP, triple superphosphate.

the kanchi treatment was probably due to mixing of rice straw fragments with the periphytons. Keshavnath, Gangadhar, Ramesh, Van Rooij, Beveredge, Baird, Verdegem and Van Dam (2001) had also reported a similar problem using sugarcane bagasse as a substrate. Rice straw had more bacteria than kanchi probably because rice straw provided more surface area. Bacteria total plate count in rice straw in the present experiment was higher than that obtained by Ramesh et al. (1999) in sugarcane bagasse, paddy straw and Eichhornia.

The two substrate treatments gave better fish yield than the control, probably due to the provision of additional foods in terms of periphyton (Miller \& Falace 2000) and bacterial biofilm (Ramesh et al. 1999). Total weight gains of rohu and catla increased significantly in the substrate treatments over the control similar to the findings of Azim, Rahman, Wahab, Asaeda, Little and Verdegem (2004). Higher total weight gain of rohu in the substrate treatments could be explained by its periphyton grazing habit (NFEP 1997). Periph- yton feeding habit of rohu benefited catla because of reduced interspecific competition. In ponds without substrates, rohu and catla tend to compete with each other for planktons (Azim et al. 2002b). Generally, catla is faster growing compared with other Indian major carp (Chakrabarty 1998). They did not grow faster in this experiment possibly due to insufficient zooplanktons in the pond water. Most probably, highly efficient grazer silver carp affected catla growth negatively through removal of zooplankton and by reducing food availability for zooplankton (Kadir, Wahab, Milstein, Hossain \& Seraji 2007). Higher daily weight gain and larger mean final size of common carp in the rice straw treatment could be attributed to high organic matter $(2.0 \%)$ deposited to the pond bottom and to its bottom-feeding habit. Substrates did not have a significant effect on the growth and production of mrigal. An earlier report also found that a periphyton-based pond polyculture system with bamboo substrates resulted in lower growth (Azim et al. 2004). Silver carp grew equally 
well in all three treatments independent of substrates.

The rice straw treatment had the highest economic return, due to the low cost of rice straw and high fish yield in the rice straw treatment. Despite the highest gross return, the kanchi treatment was less profitable than the rice straw treatment, mainly due to the relatively higher cost of kanchi. Comparatively less profit in the control was due to the lower fish yield.

The substrates (rice straw and kanchi) added to the ponds increased fish yield substantially and did not affect the water quality negatively; thus, the substrate treatments were better than the treatment without a substrate (control). Periphyton and bacterial biofilm have driven higher fish yield in the substrate treatments. Considering economics, rice straw is a cheaper substrate than kanchi. Partial harvesting is also easier in rice straw ponds because rice straw mats can be removed easily from the ponds. This simple technique has potential application in the periphyton-based rural aquaculture. As rice straw decomposes gradually, further research is needed on using rice straw as a substrate for long-term fish culture.

\section{Acknowledgments}

This research is supported by Aquaculture Collaborative Research Support Programme (ACRSP), USAID Grant No. LAG-G-00-96-90015-00, and contributions from the University of Michigan, the Asian Institute of Technology and the Bangladesh Agricultural University (BAU) at Mymensingh. The authors wish to thank Prof. M. Bahanur Rahman for guiding microbiological work, and Dr Zoardar Farukh Ahmed, Mr Abaidullah Masud, Mr Shaheen, Mr Md. Asaduzzaman, the students and staff of Central Laboratory at BAU, Dr Tek Bahadur Gurung, Asha Rayamajhi and Nita Pradhan of Fisheries Research Division, Nepal Agriculture Research Council, for their help with carrying out the research. The ACRSP accession number is 1340 .

\section{References}

APHA. (1980) Standard Methods for the Examination of Water and Wastewater, 15th edn. American Public Health Association, American Water Work Association and Water Pollution Control Federation, Washington, DC, USA.

Azim M.E., Wahab M.A., Van Dam A.A., Beveridge M.C.M. \& Verdegem M.C.J. (2001a) The potential of periphyton based culture of two Indian major carps, rohu, Labeo rohita (Hamilton) and gonia, Labeo gonius (Linnaeus). Aquaculture Research 32, 209-216.

Azim M.E., Wahab M.A., Van Dam A.A., Beveridge M.C.M., Huisman E.A. \& Verdegem M.C.J. (2001b) Optimization of stocking ratios of two Indian major carps, rohu (Labeo rohita Ham.) and catla (Catla catla Ham.) in a periphyton-based aquaculture system. Aquaculture 203, $33-49$.

Azim M.E., Verdegem M.C.J., Khatun H., Wahab M.A., Van Dam A.A. \& Beveridge M.C.M. (2002a) A comparison of fertilization, feeding and three periphyton substrates for increasing fish production in freshwater pond aquaculture in Bangladesh. Aquaculture 212, 227-243.

Azim M.E., Verdegem M.C.J., Rahman M.M., Wahab M.A., Van Dam A.A. \& Beveridge M.C.M. (2002b) Evaluation of polyculture with Indian major carps in periphyton-based pond. Aquaculture 213, 131-149.

Azim M.E., Rahman M.M.,Wahab M.A., AsaedaT., Little D.C. \& Verdegem M.C.J. (2004) Periphyton-based pond polyculture system: a bioeconomic comparison of on-farm and on-station trials. Aquaculture 242, 381-396.

Bellinger E.G. (1992) A Key to Common Algae. The Institute of Water and Environmental Management, London, UK.

Chakrabarty N.M. (1998) Biology, Culture and Production of Indian Major Carp - A Review. Narendra Publishing House, Delhi, India.

Dharmaraj M., Manissery J.K. \& Keshavnath P. (2002) Effects of a biodegradable substrate, sugarcane bagasse and supplementary feed on growth and production of fringelipped peninsular carp, Labeo fimbriatus (Bloch). Acta Ichthyologica et Piscatoria 32, 137-144.

Kadir M.A.,Wahab M.A., Milstein A., Hossain M.A. \& Seraji M.T.I., (2007) Effects of silver carp and the small indigenous fish mola Amblypharyngodon mola and punti Puntius sophore on fish polyculture production. Aquaculture 273, 520-531.

Keshavnath P., Gangadhar B., Ramesh T.J., Van Rooij J.M., Beveredge M.C.M., Baird D.J., Verdegem M.C.J. \& Van Dam A.A. (2001) Use of artificial substrates to enhance production of freshwater herbivorous fish in pond culture. Aquaculture Research 32, 189-197.

Miller M.W. \& Falace A. (2000) Evaluation methods for trophic resource factor-nutrients, primary production, and associated assemblages. In: Artificial Reef Evaluation with Application to Natural Habitats (ed. by W. Seaman Jr.), pp. 95-126. CRC Press, Florida, USA.

Moriarty D.J.W. (1997) The role of microorganisms in aquaculture ponds. Aquaculture 151, 333-349.

Mridula R.M., Manissary J.K., Keshavnath P., Shankar K.M., Nandesha M.C. \& Rajesh K.M. (2003) Water quality, biofilm production and growth of fringe-lipped carp (Labeo fimbriatus) in tanks provided with two solid substrates. Aquaculture 87, 263-267.

Mridula R.M., Manissary J.K., Keshavnath P., Shankar K.M., Nandesha M.C. \& Rajesh K.M. (2005) Effects of paddy 
straw and sugarcane bagasse on water quality, bacterial biofilm production and growth and survival of rohu, Labeo rohita (Hamilton). Aquaculture Research 36, 635-642.

Needham J.G. \& Needham P.R. (1962) A Guide to the Study of Fresh-Water Biology. Holden-Day, San Francisco, USA.

NFEP (1997) Production enhancement of the Indian major carp, Labeo rohita (hamilton) using bamboo trimmings as a substrate for the growth of periphyton. NFEP Paper No.10. Northwest Fisheries Extension Project, Parbatipur, Dinajpur, Bangladesh.

Rahman M.M., Verdegem M.C.J., Nagelkerke L.A.J., Wahab M.A., Milstein A. \& Verreth J.A.J. (2006) Growth production and food preference of rohu Labeo rohita $(\mathrm{H})$ in monoculture and in polyculture with common carp Cyprinus carpio (L.) under fed and non-fed ponds. Aquaculture 257, 359-372.

Ramesh M.R., Shankar K.M., Mohan C.V. \& Varghese T.J. (1999) Comparison of three plant substrates for enhancing carp growth through bacterial biofilm. Aquaculture Engineering 19, 119-131.
Raveh A. \& Avnimelech Y. (1979) Total Nitrogen Analysis in Water, Soil and Plant Material with Persulfate Digestion. Water Research 13, 911-912.

Van Dam A.A., Beveridge M.C.M., Azim M.E. \& Verdegem M.C.J. (2002) The potential of fish production based on periphyton. Reviews in Fish Biology and Fisheries 12, $1-31$.

Wahab M.A., Azim M.E., Ali M.H., Beveridge M.C.M. \& Khan S. (1999) The potential of periphyton based culture of the native major carp calbaush, Labeo calbasu (Hamilton). Aquaculture Research 30, 409-419.

Ward H.B. \& Whipple G.C. (1959) Freshwater Biology. John Willey and sons, New York, USA.

Wetzel R.G. (1983) Attached algal-substrata interactions; fact or myth, and when and how? In: Periphyton of Freshwater Ecosystems (ed. by R.G. Wetzel), pp. 207-215. Dr.W. Junk publishers, The Hague, the Netherlands.

Yi Y., Lin C.K. \& Diana J.S. (2003) Techniques to mitigate clay turbidity problems in fertilized earthen fish ponds. Aquaculture Engineering 27, 39-51. 